\section{Our rapidly changing understanding of acute and chronic disorders of consciousness: challenges for neurologists}

\author{
Ithabi S Gantner', Olivier Bodart', Steven Laureys' \& Athena Demertzi¹ \\ Coma Science Group, Cyclotron Research Center \& CHU Neurology Department, University of Liège, Allée \\ du 6 Août no 8, Sart Tilman B30, 4000 Liège, Belgium \\ *Author for correspondence: Tel.: +3243662303 = Fax: +3243662946 = a.demertzi@ulg.ac.be
}

A number of recent studies suggest that some 'vegetative state' patients have been misdiagnosed, judging by their ability to follow commands and in some cases even communicate through brain activity. Such studies highlight the difficulty in forming a diagnosis based only on behavioral assessments. We think that neuroimaging and electrophysiology methods will be used more frequently in clinical settings, integrated with existing behavioral assessments. Such efforts are expected to lead to a more accurate understanding of individual patients' cognitive abilities or even provide prognostic indicators. In terms of treatment planning (i.e., pain management and end-of-life decision-making), patients with disorders of consciousness are now offered the possibility of expressing their preferences by means of brain-computer interfaces. What remains to be clarified is the degree to which such indirect responses can be considered reliable and of legal representation.

It is not always easy to detect unambiguous signs of consciousness in patients with limited behavioral responses, such as coma survivors. Here, we aim to discuss the challenges that neurologists and assisting personnel are facing when dealing with patients with disorders of consciousness (DOC) in terms of diagnosis, prognosis and medical treatment. We will see that behavioral assessment remains the gold standard for diagnosing comatose, unresponsive ('vegetative') patients and patients in a minimally conscious state (MCS). Considering the persistence of diagnostic error in clinical evaluation, we highlight the need for objective biomarkers through neuroimaging and electrophysiological technologies. These technologies do not only assist clinicians in terms of differential diagnosis and potential prognosis, but also provide patients with muscle-independent means to communicate with their environment. For the moment, such technological advances remain experimental and require standardization if they are to be used in clinical routine. Importantly, clinical reality requires fast and valid assessments at the single-patient level. We foresee that an integrative approach of multimodal testing will shed light on the individual patient's pathophysiology and the underlying mechanisms. Eventually, new knowledge can lead to a redefinition of existing clinical taxonomies. Such advances are expected to lead to ethical and legal discussions, where patients' competency will potentially need to be re-established in a context of contemporary technology usage (e.g., by brain-computer interfaces), which allows them to express their wishes and even end-of-life preferences.

\section{Disorders of consciousness}

Since the 1950s and the introduction of the mechanical ventilator in intensive care, patients who would have previously died from apnea can have their respiratory functions sustained. As a result, the concept of death evolved. In the 1960s, the medical society provided the neurocentric definition of brain death as a condition of irreversible unconsciousness, clinically evidenced by the loss of all brainstem reflexes and the demonstration of continuing cessation of respiration [1]. In many cases, patients survive (brain) death, but can end up in profound states of unconsciousness. Coma, for example, is a pathological state marked by severe and prolonged dysfunction of vigilance and consciousness (at least $1 \mathrm{~h}$ ) [2]. Patients in this state show continuous absence of eye opening as well as the absence of any voluntary behavioral responses. Coma is a time-limited condition leading either to death, the recovery of consciousness or a 'vegetative state' (VS). Patients in a VS open their eyes (giving the impression of sleep-wake cycles), but they are unaware of themselves and their environment because they do not show

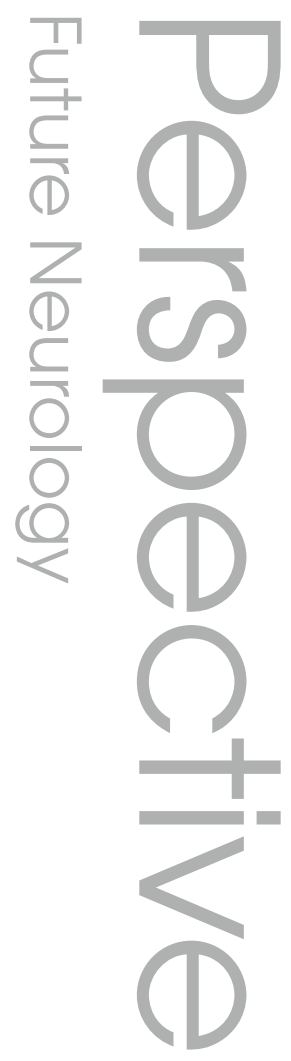

\section{Keywords}

- behavioral assessment m brain-computer interface m coma $=$ ethics $=$ fMRI minimally conscious state - unresponsive wakefulness syndrome

\section{Future : Medicine part of}


sustained, reproducible, purposeful or voluntary behavioral responses to sensory stimuli [3]. More recently, it has been recognized that some of the healthcare professionals, media and lay public feel uncomfortable using the unintended denigrating 'vegetable-like' connotation (seemingly intrinsic to the term VS). Hence, the European Task Force on Disorders of Consciousness proposed the alternative name 'unresponsive wakefulness syndrome' (UWS), a more neutral and descriptive term, pertaining to patients showing a number of clinical signs of unresponsiveness (i.e., without response to commands or oriented voluntary movements) in the presence of wakefulness [4]. Some patients will evolve from this condition to a MCS. MCS patients show inconsistent, but clearly discernible, signs of consciousness, such as command following or other purposeful behaviors (e.g., verbalizations, direct response to moving stimuli and visual pursuit) [5]. Considering the lack of a unique code for MCS, which may hamper scientific investigations, medical information retrieval, demographic and international analyses on prevalence and prognosis on DOC, we have recently proposed to subcategorize this clinical entity [6]. Mainly based on the linguistic abilities of MCS patients, and also supported by neuroanatomical data [7], we have recently subcategorized this entity as MCS+ (describing high-level behavioral responses such as command following or specific responses to linguistic content) and MCS (describing lowlevel non-reflex behavior such as visual pursuit, localization of pain or appropriate smiling to emotional stimuli) [7]. Emergence from MCS is defined by the ability of these patients to reliably communicate or use objects in a functional way. There are situations, however, where conscious patients are quadriplegic and anarthric with fully preserved cognitive abilities that they can only communicate by using the movement of their eyes or eyelids. These patients are considered to have locked-in syndrome (LIS) [8]. Acute ventral pontine lesions in the brainstem are the most common cause for LIS. As such, LIS patients do not experience disturbed consciousness. Since patients with LIS are unable to speak or move the extremities, differential diagnosis from DOC is difficult and often delayed. As a result, the diagnosis of LIS can be missed, with the consequence that these patients are mistaken for being unconscious [9]. It can happen, then, that for some LIS patients, the only evidence for preserved consciousness is their ability to communicate via assisting technologies (i.e., functional MRI [fMRI], EEG or evoked potentials). As such, we have recently suggested that these LIS patients can be considered to have 'functional LIS' [6]. In other words, the term functional LIS is proposed for patients with a dissociation between extreme behavioral motor dysfunction and the identified preserved higher cognitive functions only measurable by functional imaging techniques.

\section{Clinical assessment of patients with DOC}

Clinical experience teaches that consciousness can be reduced to two dimensions, wakefulness and awareness [10]. Wakefulness is clinically evidenced by examining the presence of spontaneous or stimulus-induced eye opening. Awareness is clinically assessed by command following or by observing non-reflex behaviors (e.g., orientation to pain). We suggest that awareness can be further reduced to awareness of the environment (or 'external awareness'), meaning the sensory or perceptual awareness of the external world, and to awareness of self (or 'internal awareness'), referring to the mental processes that do not require the mediation of external stimuli or sensory input, such as mind wandering, daydreaming, inner speech and mental imagery [11]. Due to its firstperson nature, internal awareness is clinically more ill-defined and difficult to assess at the bedside. The existing behavioral scales, which are quick to administer and widely used in clinics, mainly focus on deducing external awareness. For example, the widely used Glasgow Coma Scale scores eye, verbal and motor responses to external stimuli [12]. The Full Outline of Unresponsiveness recognizes the difficulty to assess verbal responses, especially in cases of intubated patients or patients with tracheotomy, and requires patients to show non-verbal conscious behaviors, such as eyeblinking or hand-signing to command. Thus, the Full Outline of Unresponsiveness can detect patients with LIS but cannot always differentiate UWS and MCS patients because it does not test all of the behavioral criteria required to diagnose MCS [13]. The Coma Recovery Scale - Revised is the most sensitive scale to differentiate UWS and MCS patients because it assesses auditory, visual, motor, oromotor and communication abilities next to arousal assessment, covering the diagnostic criteria for MCS [14]. Among the existing scales, the Coma Recovery Scale - Revised has been recommended as the most appropriate tool to evaluate patients with DOC [15].

To date, bedside evaluation remains the gold standard for diagnosing this clinical population. As we will discuss later, incorrect diagnosis in patients with DOC is not a rare phenomenon [16]. In order to minimize incorrect diagnostic 
evaluation through behavioral observations, neuroimaging procedures have begun to assume an adjunctive role in the diagnostic assessment of patients with DOC.

\section{Neuroimaging \& electrophysiology}

Over the years, developed technologies have made a significant impact on the assessment and management of patients with DOC. Functional neuroimaging, PET and fMRI, as well as EEG and evoked potential studies have offered the possibility of objectively approaching covert cognitive processes in patients who are otherwise incapable of intelligible or sustained behavioral expression. Such studies have used experimental protocols to assess brain function during resting state conditions and after external stimulation. These technologies further gave the opportunity to some patients to show their ability to follow simple commands by modulating their brain activity, in the absence of verbal output. By such brain activity modulation, it was even possible to communicate with some of these patients who were otherwise diagnosed as unresponsive when merely assessed with behavioral tools.

\section{Brain function in resting state \& during passive stimulation}

In resting conditions (i.e., while patients are not performing any kind of active task and/or receive no external stimulation), PET studies show that unresponsive patients are characterized by reduced global metabolism compared with healthy subjects. Interestingly, recovery from UWS does not necessarily coincide with resumption of global metabolic activity [17]. It rather seems that some areas are more important than others for conscious functions. For instance, patients with UWS show impaired metabolism in a widespread network encompassing midline (i.e., anterior cingulate/mesiofrontal and posterior cingulate cortex/precuneus) and lateral (i.e., prefrontal and posterior parietal) associative cortices, compared with healthy controls [18]. Importantly, the connection of these areas with the thalami is restored after recovery from UWS [19]. Using PET, we recently showed that UWS patients exhibit metabolic dysfunction in both external and internal awareness networks, as well as in the thalami, compared with healthy controls. On the contrary, we found that MCS patients showed dysfunction mostly in internal awareness network and thalami compared with healthy controls, which could reflect altered selfawareness in these patients, which is difficult to quantify at the bedside [20].
In resting conditions, fMRI studies show that a network of brain areas seems to play an important role in sustaining consciousness. Posterior cingulate cortex, medial prefrontal cortex and posterior parietal cortices (areas broadly known as the default mode networkDMN), show no functional connectivity in brain death $[21,22]$. Additionally, the connectivity pattern of the default mode network was found to decrease as a function of the level of consciousness, ranging from controls and patients with LIS to MCS, UWS and coma [23]. Although the value of the fMRI resting state as a differential diagnostic tool remains to be determined, the potentially prognostic value of default mode network connectivity was shown in a cohort of patients in the acute stage of coma for whom the presence of default mode network functional connectivity was paralleled to subsequent reversibility of coma [24].

In resting conditions, various EEG paradigms have made an effort to differentiate between the clinical entities of DOC. The 15-min EEG resting state acquisitions showed that UWS patients had significantly lower connectivity than MCS in the $\theta$ and $\alpha$ frequency bands [25]. Similarly, the bi-spectral index (a measure of the depth of anesthesia) was shown to discriminate between UWS and MCS patients [26]. The bi-spectral index was also positively correlated with behavioral scores of awareness at the time of testing and was associated with outcome results at 1-year post-trauma. Additionally, an EEG entropy score of 52 (value ranging from 0 to 91 , with higher scores indicating a higher consciousness level) was shown to be able to differentiate acute unconsciousness from MCS patients with $89 \%$ sensitivity and 90\% specificity. However, the prognostic value of this measure was not high and hence cannot be recommended as a prognostic tool [27]. EEG studies measuring effective connectivity also seem able to differentiate between UWS and MCS patients. Effective connectivity is a measure of the causal relationship between brain areas. One study using mismatch negativity paradigm and applying dynamic causal modeling found that the only significant difference between UWS and MCS patients was an impairment of backward connectivity from frontal to temporal cortices [28]. The prognostic value of this test, however, needs to be further validated. In addition, when EEG effective connectivity was measured after the application of transcranial magnetic stimulation, it was found that UWS patients showed a simple, local response after 
the transcranial magnetic stimulation pulses. By contrast, MCS patients showed more complex activations after the transcranial magnetic stimulation pulses, which involved distant cortical areas ipsi- and contra-lateral to the site of stimulation [29]. Other efforts have also been made to use EEG signal patterns as a prognostic tool for DOC patients. For example, it has been observed that UWS patients who made a behavioral recovery at 3-month follow-up showed higher occipital source power in the $\alpha$ band of resting EEG when compared with those who did not [30]. This implies that cortical sources of resting $\alpha$ rhythms might predict recovery in UWS patients' behavior. Taken together, resting state studies with fMRI, PET and EEG provide an appropriate paradigm to study residual brain function in patients with DOC, thanks to its simple and fast application. It should be noted, however, that in terms of differential diagnosis, no A-level recommendations can be made yet favoring a particular type of testing. Large multicentric studies are necessary to validate their diagnostic power and to determine their prognostic value.

Importantly, brain responses to external stimuli provide valuable information not only on the preserved functional (and to some degree anatomical) connectivity among distinct brain regions, but also on the nature of detected responses. Potential pain perception capacities in MCS patients are suggested by findings of cerebral correlates of pain processing in a similar network as healthy controls [31]. The activation pattern was also much more widespread than in UWS patients. The type of stimuli used also seems to make a difference in responses. Stimuli with emotional valence (e.g., an infant cries the patient's own name) induced a much more widespread activation in MCS patients compared with meaningless noise [32]. The activation pattern was comparable with that previously obtained in healthy controls. Patients with DOC also showed higher fMRI activity in the anterior cingulate cortex after to listening to their own name as compared with listening to a familiar name and this activity correlated with the behaviorally assessed level of consciousness of the patient [33]. Such results imply that selfreferential stimuli, such as one's own name, are attention grabbing, and therefore can be used in the assessment of residual brain function of these patients.

A number of studies have used event-related potentials (ERPs; averages of segments of EEG locked to the presentation of a stimulus) to assess patients with DOC. Although early 'exogenous components' (elicited within $100 \mathrm{~ms}$ after stimulus presentation) are known to persist even in unconsciousness, later 'endogenous' ERP components (e.g., P300 - i.e., showing a positive peak $300 \mathrm{~ms}$ after stimulus presentation) can be used to infer conscious cognitive processing of information [34]. In several ERP studies, the detection of the patients' own name was used in order to assess residual linguistic preservation and self-processing. When the patient's own name was presented infrequently among other names, a differential P300 was observed in LIS, MCS and UWS patients, suggesting that the auditory system was relatively preserved in response to language stimulation $[33,35]$. In another passive oddball experiment, six out of 27 patients in UWS or MCS produced a reliable P300 response to violations of prosody in non-linguistic emotional exclamations [36]. This suggests a high level of cognitive processing for auditory stimuli beyond their most basic features in patients with DOC. A direct challenge with activation studies is that, in the absence of subjective contribution from patients, a similar-to-control brain activation pattern cannot necessarily be interpreted as evidence of a conscious percept.

\section{Functional neuroimaging \& electrophysiology as tools for command following \& communication}

A willful modulation of brain activity to a certain command can be an alternative way to evidence awareness in the absence of motor output. In a collaborative study between the Universities of Cambridge (UK) and Liège (Belgium), we showed that a patient who was behaviorally diagnosed as in UWS could follow simple commands using fMRI [37]. In particular, when the patient was asked to imagine playing tennis and walking through her house, she activated the supplementary motor area and parahippocampal area, respectively. These specific activation patterns were not different from those previously observed in a cohort of healthy volunteers [38]. Since this report, similar command-following paradigms in patients with DOC have asked patients to 'look at a screen and silently name the objects as they appear' (resulting in language network activation) [39], 'move your hand' (resulting in premotor cortex activation) [40], 'imagine swimming' (resulting in supplementary motor area activation) [41] and recently also to 'focus on either the face or house of an overlaid image' [42]. 
Similarly, active paradigms have been attempted with cheaper and portable EEGbased technologies. In the case of a young comatose woman who failed to show any motor signs of conscious awareness, command following was only able to detect evoked potentials by means of EEG, therefore leading to the diagnosis of total LIS (i.e., characterized by complete immobility including all eye movements) at the intensive care unit [43]. The task was to count a target name or her own name in a list of other names. A previous study using this task demonstrated that while most MCS patients exhibited increased amplitude of the P300 when instructed to count, no taskrelated P300 changes were observed in UWS patients [44]. Similarly, conscious processing was detected in three out of four MCS patients who were instructed to actively count the number of deviant trials in a series of sound, while no responses were recorded in UWS patients [45]. A number of studies have demonstrated that EEG power spectral analysis can be used as a flexible bedside tool to demonstrate awareness in braininjured patients who are otherwise unable to communicate [46-48].

These command-following paradigms have been further developed in order to be used as communication systems. In these studies, differentiation between 'brain responses' was used as proxy for behavioral responses. Using the same mental imagery paradigm [37], Monti et al. demonstrated that a DOC patient was able to use the modulation of his brain activity to reliably answer 'yes' or 'no' to simple questions, even though no signs of communication had ever been found through bedside examination [49]. As noted above, EEG was used with similar motor imagery paradigms, with the advantage of being cheaper and possible to use at the patient's bedside [50,51]. Using an auditory P300-based brain-computer interface, command following was accurately detected in healthy volunteers, and the same technique was then applied to establish brain-computer interface-based communication with healthy controls $[52,53]$. Accuracy of this technique with patients in MCS is still lower than for controls, but several ways to improve it are being studied. Finally, brain-computer interface applications in UWS patients are not clear and need to be evidence based.

It should be stressed that when there is no brain activation after a command to perform a mental imagery task, this does not allow strong claims about the absence of consciousness. Many possible causes could indeed account for negative responses. Many possible causes could lead to negative outcomes. Corrupted signals due to movement or other artifacts are often encountered in these patients and are particularly troublesome in fMRI experiments [54]. Indeed, in many cases patients can show increased motion in the scanner, which can hinder their evaluation on fMRI paradigms. In these cases, patients will need to be scanned under sedation or anesthesia. Therefore, only fMRI resting state acquisitions can be performed. The future challenge of this practice is twofold: first, to determine whether increased motion is in any way correlated to higher awareness levels; and second, to determine what kind of residual cognition is measured under such a sedated condition. Patient-dependent fluctuations could also be encountered due to their level of arousal (spontaneous or medication related). Lastly, since the discussed mental imagery, motor or attentional tasks require preservation of different cognitive processes (such as visual, auditory, language and working memory functions), perceptual, sensory or cognitive insufficiencies might also hamper positive outcomes. However, while negative results do not necessarily reflect proof of absence of consciousness, positive results are informative and relatively easy to interpret as a proof of consciousness. The neural responses required for these types of tasks are not produced automatically by the eliciting stimulus, but rather, depend on timedependent and sustained responses generated by the participant, and can therefore be used as a neural proxy of behavior for identifying conscious awareness. On the contrary, unconscious mental representations are usually observed in clinical and experimental contexts to be fleeting, lasting only a few seconds or less $[55,56]$. In any case, in order to minimize false positives and to enhance our understanding of the interaction between brain and behavior, we suggest that future investigations take the correlation between behavioral scores and neuroimaging data into account, which has been previously suggested [57] and performed [20,58].

\section{Challenges for clinical practice}

The evaluation of severely brain-damaged patients is extremely challenging and if one relies merely on behavioral responses when evaluating remnants of conscious behavior, they can be misled. Erroneous diagnosis in behavioral examination can have various sources. The patient might have limited physical capacities (e.g., generalized hypotonus, 
spasticity or paralysis) or cognitive function (e.g., aphasia, apraxia, agnosia), fluctuations in arousal level, fatigue, subclinical seizure activity, occult illness, pain or cortical sensory deficits (e.g., cortical blindness/deafness). Sedating medications, restricted range of movement stemming from restraints and immobilization techniques, poor positioning and excessive ambient noise, heat or light can decrease or distort voluntary behavioral responses [59]. Diagnostic accuracy might be diminished further if the range of behaviors sampled is too narrow, response time-windows are over- or under-inclusive and examinations are conducted too infrequently to capture the full range of behavioral fluctuation [16]. Error in diagnosis with patients with DOC can have serious consequences in clinical management, from treatment of pain to end-of life decisions. As a consequence, it has been estimated that approximately $40 \%$ of patients with DOC are erroneously given the clinical consensus diagnosis of UWS $[60,61]$. We recently showed that, despite the introduction of the clinical criteria for the MCS, this diagnostic error rate has not substantially changed since the 1990s [16]. As we discussed above, with the progressive use of paraclinical technologies, we can now detect subclinical signs of awareness, otherwise not evidenced at the bedside [49].

For clinicians, prognosis and the prediction of clinical outcome are among the most challenging issues when they face patients' caregivers and/or hospitals' resources. According to the Multi-Society Task Force on persistent VS, unresponsive patients of traumatic etiology show better improvement up to 12 months after injury compared with UWS patients of non-traumatic etiology [62]. It should be noted that this work was conducted before the definition and diagnostic criteria for MCS. Hence, it is likely that the studied cohort of patients could have been composed of both UWS and MCS patients. We now know that these two clinical entities are not characterized by the same clinical outcome. Indeed, it has been shown that $50 \%$ of all MCS patients progress to moderate to no disability 12 months after injury, whereas less than 5\% of UWS patients achieve this level of improvement [63]. It has also been shown that the rate of recovery in the acute setting is correlated with a better chance of recovery and better outcome [64]. Nevertheless, little is known about longterm prognosis and outcome in these patients. Even if late recovery ( $>3$ months post-injury) of patients in non-traumatic UWS is unusual, it has been documented, albeit with very poor functional outcome [65]. A number of highly publicized patients, like the case of Terry Wallis who was considered to be in a VS, made the headlines when he started to speak 19 years after his car accident [66]. We should note that such cases appear sporadically in the academic literature and do not suffice to change the clinical taxonomy of VS/UWS. Rather, they highlight the unfortunate connotation of a refutable clinical evolution linked to the names of VS/UWS. We think that larger studies on clinical outcome including information on patients' anatomical and functional lesions will eventually clarify the underlying mechanisms of fast and late recoveries. In MCS patients, late recovery seems to be more frequent. Up to $30 \%$ of patients in MCS can emerge from this stage after more than 1 year spent as minimally conscious. The functional outcome, however, remains very poor for these patients and most of them need to remain institutionalized [67].

Paraclinical techniques have also been used for prognostic purposes. So far, ERPs are used extensively in intensive care to predict clinical outcome. A meta-analysis has demonstrated that the mismatch negativity (MMN; an early negative component of the auditory ERP elicited by a deviant tone in the repetitive stimuli and thus thought to reflect sound discrimination) and the P300 component can predict awakening from coma [68]. Another study has also highlighted the predictive value for the N100 component (a negative component of auditory ERP indicating some level of processing at the sensory cortex level) [69]. A decision tree-based classification has shown that MMN, pupillary light reflex and somatosensory-evoked potentials can be used to accurately predict a significant chance of awakening (if MMN is present) or the probability of non-awakening (if $\mathrm{MMN}$ and either somatosensory-evoked potentials or pupillary light reflex are absent) [70]. Using fMRI, stimuli with emotional valence such as infant cries [32] or the patient's own name [71] can also be informative of patients' prognosis. Indeed, it was shown that two patients with UWS who showed widespread activation after listening to their own name subsequently showed clinical improvement to MCS observed 3 months after their fMRI scan [72].

For some investigators, efforts should be focused on refining prognostic methods rather than focusing on differential diagnosis [73]. We think, however, that proper decision-making 
can only be made once a valid diagnosis is established. For example, we have shown that opinions about clinical diagnosis influence views on pain perception. In a wide European survey among clinicians, the majority of participants agreed more with pain perception in MCS compared with UWS patients [74]. Similarly, a decision about treatment withdrawal was supported more for UWS and significantly less for MCS patients [75]. Finally, we also showed that for UWS patients, treatment limitation was supported more when participants recognized pain perception in these patients (FIgUre 1). These data suggest that medico-ethical controversies still exist for UWS and are influenced by personal characteristics (e.g., profession, religious beliefs and region of origin). As personal opinions can mediate medical practice [76] we think that treatment guidelines should take clinicians' attitudes into account in future formulations [77].

For several decades, researchers and clinicians have tried to identify potential means to promote arousal and awareness in patients with DOC. Medical treatments can vary from preventing infections (with nutrition and tendon retraction) to more invasive interventions with pharmacological or surgical procedures. In terms of pharmacological interventions, aminergic and neurostimulant agents seem to be the most promising for patients with DOC. Zolpidem (a non-benzodiazepine sedative drug that is used for insomnia in healthy individuals) was shown to be effective to promote arousal and awareness, albeit in a small proportion of the patients [78]. The paradoxical effect of this sleeping pill was first shown in 2000 [79] and was since then verified and tested on larger patient groups [78-80]. The current hypothesis suggests that zolpidem acts by inducing a direct inhibition of the globus pallidus (an inhibitory structure of the brain), thus liberating the corticothalamic pathway leading to frontal cortices [81]. Amantadine (known for its dopaminergic and NMDA agonist effects) was only recently studied in a randomized controlled trial [82]. The drug was shown to be highly effective in promoting arousal and awareness in MCS patients following traumatic accidents.

Painkillers are also often given as treatment for patients with DOC. Nevertheless, even for this basic treatment, a number of problems arise. First of all, assessing the feeling of pain in non-communicating patients is challenging because pain is a subjective sensation [77]. As such, patients with DOC by definition cannot provide self-ratings for feelings of pain. We have, therefore, introduced the Nociception Coma Scale to behaviorally assess painful sensations in DOC patients in a standardized way [83]. Initially comprised of four different subscales, now with three in the revised version, it allows the clinician to evaluate whether there is a need to adapt the analgesic treatment [84]. Second, although we know that UWS and MCS patients do not process painful stimuli the same way, an absent response to pain cannot be considered as absence of pain perception. Using neuroimaging, we have shown that there are differences in the way UWS and MCS patients process painful stimulation. Patients in UWS do not process noxious stimuli further than in the primary sensory cortex, preventing this sensation from being regarded as painful [85]. As patients in MCS show additional activation of high-order associative cortices (similar to healthy controls), we assume pain perception [31]. To date, it remains unclear whether pain should or should not be treated the same way in all DOC patients [86]. In light of an incomplete understanding of pain and suffering in these states, we think that pain should be closely monitored in non-communicating patients, and treated carefully.

In terms of nonpharmacological interventions, deep brain stimulation of the thalamus showed

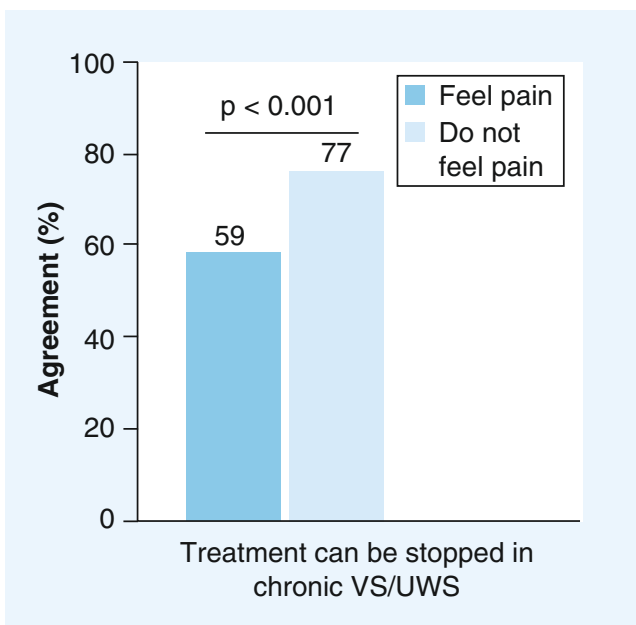

Figure 1. The ascription of pain perception in unresponsive patients correlates with opinions on treatment limitation in this patient population. In a wide European survey among clinicians, the more respondents who recognized pain perception in patients in a VS/UWS, the less they agreed with treatment withdrawal in this condition.

UWS: Unresponsive wakefulness syndrome; VS: Vegetative state.

Adapted with permission from [77]. 
behavioral improvements in a MCS patient, 6 years after the traumatic accident [87]. This technique is hypothesized to work by promoting activation of large preserved but disconnected thalamocortical networks. Despite its promising therapeutic application, deep brain stimulation of the thalamus in non-communicating patients needs to be further standardized in a larger cohort of patients of various etiologies. Lastly, non-invasive sensory stimulations (including several paradigms such as environmental enhancement and tactile stimulations) have been tested under the hypothesis that increasing brain inputs would promote neuroplasticity and hence patients would achieve a better outcome [88]. Likewise, these techniques lack evidencebased recommendations.

\section{Conclusion \& future perspective}

In the last decade, we have viewed the development and the validation of standardized behavioral scales and neuroimaging/EEG techniques to better understand patients with DOC. The number of published scientific articles seems to rise exponentially, with more than 2000 articles published in last decade (Figure 2). Such scientific advances have led, at least to a certain degree, to new subdivisions of existing diagnostic entities (i.e., MCS \pm ). We expect that with continuous scientific advances in the research of patients with DOC, further subdivisions could arise. At first glance, changes in clinical taxonomy might seem to complicate the diagnostic picture around DOC patients. However, we think that new knowledge coming from assisting technologies will need to be incorporated in the existing clinical diagnostic procedures when accessibly possible. It is true that in many cases many neuroimaging technologies are not widely accessible for clinical research. This can either be owing to high costs or as a result of simultaneous machine use by several other disciplines. In addition, the statistical analysis of such data may require substantial training. Similarly, moving from group-level to single patient-level analysis is statistically challenging but clinically salient. In the end, it is of great importance to be able to differentiate DOC patients based on their brain data for clinical practice. So far, studies have focused on how patient groups in different clinical entities differ among each other as a function of decreased consciousness level [23]. We think that with the introduction of automated statistical classifiers, the ability of neuroimaging and electrophysiological techniques to differentiate UWS, MCS \pm , emerged MCS and functional LIS patients will be enhanced. We have shown, for instance, that a classifier trained on healthy controls, PET

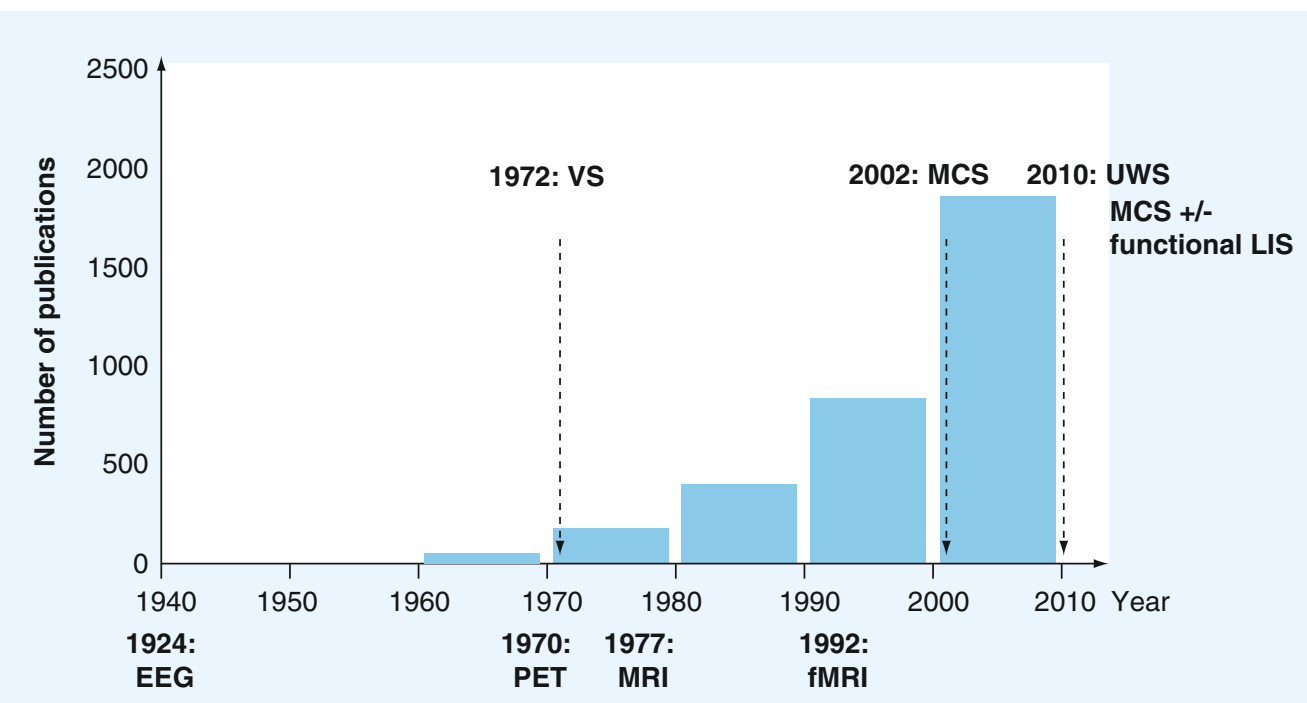

Figure 2. With the passage of time and the rise in application of new technologies for brain function assessment (as evidenced by the number of published studies), the nosology of disorders of consciousness also evolved. Although this pattern is not necessarily causal, it nevertheless shows the significant contribution of paraclinical assessment in promoting our understanding on residual cognition in patients with disorders of consciousness. Online research performed on Pubmed with the terms "EEG", "PET", "MRI" or "fMRI" and "coma", "vegetative state" and "minimally conscious state", as keywords on a time span of 10 years each time from 1940 to 2012. fMRI: Functional MRI; LIS: Locked-in syndrome; MCS: Minimally conscious state; UWS: Unresponsive wakefulness syndrome; VS: Vegetative state. 
data could classify conscious subjects from unconscious patients with $100 \%$ classification accuracy; all LIS patients were also classified as 'conscious' [89]. For some investigators, however, efforts should not be focused necessarily on differential diagnosis, but rather on refining prognostic methods [73]. We think that only with an accurate and valid diagnosis can we proceed with outcome estimation and design effective treatments. Indeed, clinical prognosis requires the study of a large cohort of patients evaluated with standardized assessment tools (e.g., with the Coma Recovery Scale - Revised). Currently, it is not well established as to what a good outcome for patients with DOC is. Should one, for instance, set the threshold of good outcome to functional recovery and social integration? If positive, then patients evolving to LIS are not considered as recovered patients. Furthermore, can we determine a specific time point after which we are certain at a $\mathrm{p}=0.05$ level that this patient is not expected to recover any more? How do we deal with late recoveries from UWS [65]? Future multicentric studies are expected to shed more light on the spectrum of consciousness and provide sensitivity and specificity values regarding clinical outcome.

Aside from the diagnostic and prognostic value of neuroimaging and EEG-based tools, these technologies are beginning to be used as alternative means for command following and communication. We think that in the future such means will need to be further validated as an appropriate way for some patients to express their wishes. What remains to be shown is whether such technologies can be used as evidence of the expressed will of a competent patient. For example, how can a negative response of an 'unresponsive' patient to the question of whether they want to continue to live can be considered as a reliable response to be respected? Similarly, should pain treatment in a MCS patient change once they communicate that they suffer? Should proving consciousness in these patients be considered as piece of evidence to be celebrated, or can it work against patients' and families' best interests? These aforementioned questions require answers that future establishment of ethical and legal provisions can provide. To date, the US law authorizes withdrawal of nutrition and hydration without analgesics, if these patients have been stable for more than 12 months in the case of traumatic etiology and 3 months for anoxic etiology [90]. The problem is that this decision is based on a third person view; it is a decision they would make for their patients and not for themselves [75]. We would like to mention that here we do not aim to directly change the law based on such sporadic clinical

\section{Executive summary}

\section{Disorders of consciousness}

- The recent re-definition of vegetative state as unresponsive wakefulness syndrome, the subcategorization of minimally conscious state (MCS) into MCS + and MCS- and the introduction of functional locked in syndrome enrich the spectrum of disorders of consciousness.

\section{Clinical assessment of patients with disorders of consciousness}

- Standardized behavioral evaluation of consciousness must be used for its clinical assessment.

- Although the Coma Recovery Scale - Revised is the most recommended scale, deficiencies in criterion validity and diagnostic validity need to be targeted in future research.

\section{Neuroimaging}

- Resting state studies with neuroimaging technologies have shown consciousness to be an emergent property of widespread thalamo-corticalfrontoparietal network connectivity.

- Cognitive processing assessed in passive stimulation studies can provide valuable prognostic and diagnostic information.

- Novel imaging paradigms could show command following in behaviorally unresponsive patients.

Functional MRI and electroencephalography-based brain-computer interfaces for two-way communication with some behaviorally unresponsive patients have been established and more sophisticated communicative bedside devices are being developed.

\section{Challenges for clinical practice}

- Prognosis remains to be further studied in the new entities of disorders of consciousness. Outcome and recovery are influenced by diagnosis, etiology and time spent in a disorder of consciousness.

- Promising pharmacologic (zolpidem and amantadine) and non-pharmacologic (deep brain stimulation) treatments should be tested in large-scale double-blind randomized clinical trials.

Pain management can be improved by using the Nociception Coma Scale - Revised.

End-of-life decisions and other ethical issues could be probed in the future through brain-computer interface communication; however, using such indirect means of communication for such grave questions remains controversial. 
cases. We rather propose the introduction of supplementary legal provisions that will also provide medico-legal cover for those few patients showing atypical ways of communication with their surroundings.

Considering the above, we expect that in the coming 10 years, a drop in the misdiagnosis rate will be observed. With a wider use of standardized scales, the development of multimodal neuroimaging techniques and of bedside EEG evaluation, correct and finer diagnosis will be easier to make. We also expect these methods to help clinicians and other caregivers understand the state of DOC of their patients. Among the future challenges is the application of such methodologies at the singlesubject level as clinical reality requires.

Financial \& competing interests disclosure

This work was supported by the Belgian National Funds for Scientific Research, the European Commission, the James McDonnell Foundation, the European Space Agency, Mind Science Foundation, the French Speaking Community Concerted Research Action (ARC-06/11-340), the Public Utility Foundation "Université Européenne du Travail", "Fondazione Europea di Ricerca Biomedica" and the University and University Hospital of Liège (Belgium). The authors have no other relevant affliations or financial involvement with any organization or entity with a financial interest in or financial conflict with the subject matter or materials discussed in the manuscript apart from those disclosed.

No writing assistance was utilized in the production of this manuscript.

\section{References}

Papers of special note have been highlighted as:

- of interest

.. of considerable interest

1. Wijdicks EF. The diagnosis of brain death. N. Engl. J. Med. 344(16), 1215-1221 (2001).

2. Plum F, Posner JB. The Diagnosis of Stupor and Coma. FA Davis Co., PA, USA (1966).

3. Monti MM, Laureys S, Owen AM. The vegetative state. BMJ 341, c3765 (2010).

4. Laureys S, Celesia GG, Cohadon F et al. Unresponsive wakefulness syndrome: a new name for the vegetative state or apallic syndrome. BMC Med. 8, 68 (2010).

- The European Task Force on Disorders of Consciousness proposes an alternative name for the 'vegetative state' to overcome the persistently negative connotation attached to the term.

5. Giacino JT, Ashwal S, Childs $\mathrm{N}$ et al. The minimally conscious state. Neurology 58(3), 349-353 (2002).

6. Bruno MA, Vanhaudenhuyse A, Thibaut A, Moonen G, Laureys S. From unresponsive wakefulness to minimally conscious PLUS and functional locked-in syndromes: recent advances in our understanding of disorders of consciousness. J. Neurol. 258(7), 1373-1384 (2011).

7. Bruno MA, Majerus S, Boly M et al. Functional neuroanatomy underlying the clinical subcategorization of minimally conscious state patients. J. Neurol. 259(6), 1087-1098 (2012).

8. Recommendations for use of uniform nomenclature pertinent to patients with severe alterations in consciousness. American Congress of Rehabilitation Medicine. Arch. Phys. Med. Rehabil. 76(2), 205-209 (1995).
9. Laureys S, Pellas F, Van Eeckhout P et al. The locked-in syndrome: what is it like to be conscious but paralyzed and voiceless? Prog. Brain Res. 150, 495-511 (2005).

10. Posner JB, Saper CB, Schiff ND, Plum F. Plum and Posner's Diagnosis of Stupor and Coma. Oxford University Press, Oxford, UK (2007).

11. Vanhaudenhuyse A, Demertzi A, Schabus M et al. Two distinct neuronal networks mediate the awareness of environment and of self. J. Cogn. Neurosci. 23(3), 570-578 (2011).

12. Teasdale G, Jennett B. Assessment of coma and impaired consciousness. A practical scale. Lancet 2 (7872), 81-84 (1974).

13. Schnakers C, Giacino J, Kalmar K et al. Does the FOUR score correctly diagnose the vegetative and minimally conscious states? Ann. Neurol. 60(6), 744-745 (2006).

14. Giacino JT, Kalmar K, Whyte J. The JFK coma recovery scale-revised: measurement characteristics and diagnostic utility. Arch. Phys. Med. Rehabil. 85(12), 2020-2029 (2004).

15. Seel RT, Sherer M, Whyte J et al. Assessment scales for disorders of consciousness: evidencebased recommendations for clinical practice and research. Arch. Phys. Med. Rehabil. 91(12), 1795-1813 (2010).

- First extensive, systematic and evidencebased review of 13 behavioral assessment scales for patients with disorders of consciousness.

16. Schnakers C, Vanhaudenhuyse A, Giacino J et al. Diagnostic accuracy of the vegetative and minimally conscious state: clinical consensus versus standardized neurobehavioral assessment. BMC Neurol. 9, 35 (2009).

- Milestone study confirming the unchanged high misdiagnosis rate in vegetative state and demonstrating the usefulness of standardized validated behavioral assessment tools.

17. Laureys S, Owen AM, Schiff ND. Brain function in coma, vegetative state, and related disorders. Lancet Neurol. 3(9), 537-546 (2004).

18. Laureys S, Goldman S, Phillips C et al. Impaired effective cortical connectivity in vegetative state: preliminary investigation using PET. NeuroImage 9(4), 377-382 (1999).

19. Laureys S, Faymonville ME, Luxen A, Lamy M, Franck G, Maquet P. Restoration of thalamocortical connectivity after recovery from persistent vegetative state. Lancet 355(9217), 1790-1791 (2000).

20. Thibaut A, Bruno MA, Chatelle C et al. Metabolic activity in external and internal awareness networks in severely braindamaged patients. J. Rehabil. Med. 44(6), 487-494 (2012).

21. Boly M, Tshibanda L, Vanhaudenhuyse A et al. Functional connectivity in the default network during resting state is preserved in a vegetative but not in a brain dead patient. Hum. Brain Mapp. 30 (8), 2393-2400 (2009).

22. Soddu A, Vanhaudenhuyse A, Demertzi A et al. Resting state activity in patients with disorders of consciousness. Funct. Neurol. 26(1), 37-43 (2011).

23. Vanhaudenhuyse A, Noirhomme Q, Tshibanda LJ et al. Default network connectivity reflects the level of consciousness in non-communicative brain-damaged patients. Brain 133(Pt 1), 161-171 (2010).

-. Shows the potential diagnostic value of recording the brain's activity without any stimulation or task, providing a strong 
link between connectivity and consciousness.

24. Norton L, Hutchison RM, Young GB, Lee DH, Sharpe MD, Mirsattari SM. Disruptions of functional connectivity in the default mode network of comatose patients. Neurology 78(3), 175-181 (2012)

25. Lehembre R, Marie-Aurelie B, Vanhaudenhuyse A et al. Resting-state EEG study of comatose patients: a connectivity and frequency analysis to find differences between vegetative and minimally conscious states. Funct. Neurol. 27(1), 41-47 (2012).

26. Schnakers C, Ledoux D, Majerus S et al. Diagnostic and prognostic use of bispectral index in coma, vegetative state and related disorders. Brain Inj. 22(12), 926-931 (2008).

27. Gosseries O, Schnakers C, Ledoux D et al. Automated EEG entropy measurements in coma, vegetative state/unresponsive wakefulness syndrome and minimally conscious state. Funct. Neurol. 26(1), 25-30 (2011).

28. Boly M, Garrido MI, Gosseries O et al. Preserved feedforward but impaired top-down processes in the vegetative state. Science 332(6031), 858-862 (2011).

29. Rosanova M, Gosseries O, Casarotto S et al. Recovery of cortical effective connectivity and recovery of consciousness in vegetative patients. Brain 135(Pt 4), 1308-1320 (2012).

30. Babiloni C, Sara M, Vecchio F et al. Cortical sources of resting-state $\alpha$ rhythms are abnormal in persistent vegetative state patients. Clin. Neurophysiol. 120(4), 719-729 (2009).

31. Boly M, Faymonville ME, Schnakers C et al. Perception of pain in the minimally conscious state with PET activation: an observational study. Lancet Neurol. 7(11), 1013-1020 (2008).

32. Laureys S, Perrin F, Faymonville ME et al. Cerebral processing in the minimally conscious state. Neurology 63(5), 916-918 (2004).

33. Qin P, Di H, Yan X et al. Mismatch negativity to the patient's own name in chronic disorders of consciousness. Neurosci. Lett. 448(1), 24-28 (2008).

34. Vanhaudenhuyse A, Laureys S, Perrin F. Cognitive event-related potentials in comatose and post-comatose states. Neurocrit. Care 8(2), 262-270 (2008).

35. Perrin F, Schnakers C, Schabus M et al. Brain response to one's own name in vegetative state, minimally conscious state, and lockedin syndrome. Arch. Neurol. 63(4), 562-569 (2006).

36. Kotchoubey B, Kaiser J, Bostanov V, Lutzenberger W, Birbaumer N. Recognition of affective prosody in brain-damaged patients and healthy controls: a neurophysiological study using EEG and whole-head MEG. Cogn. Affect. Behav. Neurosci. 9(2), 153-167 (2009).

37. Owen AM, Coleman MR, Boly M, Davis MH, Laureys S, Pickard JD. Detecting awareness in the vegetative state. Science 313(5792), 1402 (2006).

38. Boly M, Coleman MR, Davis MH et al. When thoughts become action: an fMRI paradigm to study volitional brain activity in non-communicative brain injured patients. NeuroImage 36(3), 979-992 (2007).

39. Rodriguez Moreno D, Schiff ND, Giacino J, Kalmar K, Hirsch J. A network approach to assessing cognition in disorders of consciousness. Neurology 75(21), 1871-1878 (2010).

40. Bekinschtein TA, Manes FF, Villarreal M, Owen AM, Della-Maggiore V. Functional imaging reveals movement preparatory activity in the vegetative state. Front. Hum. Neurosci. 5, 5 (2011).

41. Bardin JC, Fins JJ, Katz DI et al. Dissociations between behavioural and functional magnetic resonance imagingbased evaluations of cognitive function after brain injury. Brain 134(Pt 3), 769-782 (2011).

42. Monti MM, Pickard JD, Owen AM. Visual cognition in disorders of consciousness: from V1 to top-down attention. Hum. Brain Mapp. doi:10.1002/hbm.21507 (2012) (Epub ahead of print).

43. Bauer G, Gerstenbrand F, Rumpl E. Varieties of the locked-in syndrome. J. Neurol. 221(2), 77-91 (1979).

44. Schnakers C, Perrin F, Schabus M et al. Voluntary brain processing in disorders of consciousness. Neurology 71(20), 1614-1620 (2008).

45. Bekinschtein TA, Dehaene S, Rohaut B, Tadel F, Cohen L, Naccache L. Neural signature of the conscious processing of auditory regularities. Proc. Natl Acad. Sci. USA 106(5), 1672-1677 (2009).

46. Cruse D, Owen AM. Consciousness revealed: new insights into the vegetative and minimally conscious states. Curr. Opin. Neurol. 23(6), 656-660 (2010).

47. Cruse D, Chennu S, Chatelle C et al. Bedside detection of awareness in the vegetative state: a cohort study. Lancet 378(9809), 2088-2094 (2011).

48. Goldfine AM, Victor JD, Conte MM, Bardin JC, Schiff ND. Determination of awareness in patients with severe brain injury using EEG power spectral analysis. Clin. Neurophysiol. 122(11), 2157-2168 (2011).
49. Monti MM, Vanhaudenhuyse A, Coleman MR et al. Willful modulation of brain activity in disorders of consciousness. N. Engl. J. Med. 362(7), 579-589 (2010).

- Milestone article on the potential of functional MRI for two-way communication with a behaviorally unresponsive patient. It highlights how much more there is to learn about the vegetative and minimally conscious states and opens the possibility for more sophisticated communicative devices in the future.

50. Birbaumer N, Murguialday AR, Cohen L. Brain-computer interface in paralysis. Curr. Opin. Neurol. 21(6), 634-638 (2008).

51. Kotchoubey B, Lang S, Winter S, Birbaumer N. Cognitive processing in completely paralyzed patients with amyotrophic lateral sclerosis. Eur. J. Neurol. 10(5), 551-558 (2003).

52. Lule D, Noirhomme Q, Kleih SC et al. Probing command following in patients with disorders of consciousness using a brain-computer interface. Clin. Neurophysiol. doi:10.1016/j.clinph.2012.04.030 (2012) (Epub ahead of print).

53. Chatelle C, Chennu S, Noirhomme Q, Cruse D, Owen AM, Laureys S. Brain-computer interfacing in disorders of consciousness. Brain Inj. 26(12), 1510-1522 (2012).

54. Soddu A, Vanhaudenhuyse A, Bahri MA et al. Identifying the default-mode component in spatial IC analyses of patients with disorders of consciousness. Hum. Brain Mapp. 33(4), 778-796 (2012).

55. Greenwald AG, Draine SC, Abrams RL. Three cognitive markers of unconscious semantic activation. Science 273(5282), 1699-1702 (1996).

56. Naccache L. Psychology. Is she conscious? Science 313(5792), 1395-1396 (2006).

57. Fox MD, Greicius M. Clinical applications of resting state functional connectivity. Front. Syst. Neurosci. 4, 19 (2010).

58. Bruno MA, Vanhaudenhuyse A, Schnakers C et al. Visual fixation in the vegetative state: an observational case series PET study. BMC Neurol. 10, 35 (2010).

59. Giacino JT, Schnakers C, Rodriguez-Moreno D, Kalmar K, Schiff N, Hirsch J. Behavioral assessment in patients with disorders of consciousness: gold standard or fool's gold? Prog. Brain Res. 177, 33-48 (2009).

60. Andrews K, Murphy L, Munday R, Littlewood C. Misdiagnosis of the vegetative state: retrospective study in a rehabilitation unit. BMJ 313(7048), 13-16 (1996).

61. Childs NL, Mercer WN, Childs HW. Accuracy of diagnosis of persistent vegetative state. Neurology 43(8), 1465-1467 (1993). 
62. Medical aspects of the persistent vegetative state (2). The Multi-Society Task Force on PVS. N. Engl. J. Med. 330(22), 1572-1579 (1994).

63. Giacino J, Kalmar K. The vegetative and minimally conscious states: a comparison of clinical features and functional outcome. J. Head Traum. Rehabil. 12(4), 36-51 (1997).

64. Whyte J, Gosseries O, Chervoneva I et al. Predictors of short-term outcome in braininjured patients with disorders of consciousness. Prog. Brain Res. 177, 63-72 (2009).

65. Estraneo A, Moretta P, Loreto V, Lanzillo B, Santoro L, Trojano L. Late recovery after traumatic, anoxic, or hemorrhagic longlasting vegetative state. Neurology 75(3), 239-245 (2010)

66. Voss HU, Uluc AM, Dyke JP et al. Possible axonal regrowth in late recovery from the minimally conscious state. J. Clin. Invest. 116(7), 2005-2011 (2006).

67. Luaute J, Maucort-Boulch D, Tell L et al. Long-term outcomes of chronic minimally conscious and vegetative states. Neurology 75(3), 246-252 (2010).

- Provides helpful information about longterm prognosis in unresponsive wakefulness syndrome and minimally conscious state patients. They were among the first to study a reasonable amount of patients with each diagnosis for such a long time (5 years).

68. Daltrozzo J, Wioland N, Mutschler V, Kotchoubey B. Predicting coma and other low responsive patients outcome using eventrelated brain potentials: a meta-analysis. Clin. Neurophysiol. 118(3), 606-614 (2007).

69. Luaute J, Fischer C, Adeleine P, Morlet D, Tell L, Boisson D. Late auditory and eventrelated potentials can be useful to predict good functional outcome after coma. Arch. Phys. Med. Rehabil. 86(5), 917-923 (2005).

70. Fischer C, Luaute J, Nemoz C, Morlet D, Kirkorian G, Mauguiere F. Improved prediction of awakening or nonawakening from severe anoxic coma using tree-based classification analysis. Crit. Care Med. 34(5), 1520-1524 (2006)

71. Di HB, Yu SM, Weng XC et al. Cerebral response to patient's own name in the vegetative and minimally conscious states. Neurology 68(12), 895-899 (2007).

72. Di H, Boly M, Weng X, Ledoux D, Laureys $S$. Neuroimaging activation studies in the vegetative state: predictors of recovery? Clin. Med. 8(5), 502-507 (2008).

73. Sara M, Pistoia F. Bedside detection of awareness in the vegetative state. Lancet 379(9827), 1702-1703 (2012).

74. Demertzi A, Schnakers C, Ledoux D et al. Different beliefs about pain perception in the vegetative and minimally conscious states: a European survey of medical and paramedical professionals. Prog. Brain Res. 177, 329-338 (2009).

75. Demertzi A, Ledoux D, Bruno MA et al. Attitudes towards end-of-life issues in disorders of consciousness: a European survey. J. Neurol. 258(6), 1058-1065 (2011).

76. Cabana MD, Rand CS, Powe NR et al. Why don't physicians follow clinical practice guidelines? A framework for improvement. JAMA 282(15), 1458-1465 (1999).

77. Demertzi A, Racine E, Bruno M et al. Pain perception in disorders of consciousness: neuroscience, clinical care, and ethics in dialogue. Neuroethics doi:0.1007/s12152-0119149-x (2012) (Epub ahead of print).

78. Whyte J, Myers R. Incidence of clinically significant responses to zolpidem among patients with disorders of consciousness: a preliminary placebo controlled trial. Am. J. Phys. Med. Rehabil. 88(5), 410-418 (2009).

79. Clauss RP, Guldenpfennig WM, Nel HW, Sathekge MM, Venkannagari RR. Extraordinary arousal from semi-comatose state on zolpidem. A case report. S. Afr. Med. J. 90(1), 68-72 (2000).
80. Brefel-Courbon C, Payoux P, Ory F et al. Clinical and imaging evidence of zolpidem effect in hypoxic encephalopathy. Ann. Neurol. 62(1), 102-105 (2007).

81. Brown EN, Lydic R, Schiff ND. General anesthesia, sleep, and coma. N. Engl. J. Med. 363(27), 2638-2650 (2010).

82. Giacino JT, Whyte J, Bagiella E et al. Placebo-controlled trial of amantadine for severe traumatic brain injury. N. Engl. J. Med. 366(9), 819-826 (2012).

83. Schnakers C, Chatelle C, Vanhaudenhuyse A et al. The nociception coma scale: a new tool to assess nociception in disorders of consciousness. Pain 148(2), 215-219 (2010).

84. Chatelle C, Majerus S, Whyte J, Laureys S, Schnakers C. A sensitive scale to assess nociceptive pain in patients with disorders of consciousness. J. Neurol. Neurosurg. Psychiatry 83(12), 1233-1237 (2012).

85. Laureys S, Faymonville ME, Peigneux $\mathrm{P}$ et al. Cortical processing of noxious somatosensory stimuli in the persistent vegetative state. NeuroImage 17(2), 732-741 (2002).

86. Schnakers C, Chatelle C, Demertzi A, Majerus S, Laureys $\mathrm{S}$. What about pain in disorders of consciousness? AAPS J. 14(3), 437-444 (2012).

87. Schiff ND, Giacino JT, Kalmar K et al. Behavioural improvements with thalamic stimulation after severe traumatic brain injury. Nature 448(7153), 600-603 (2007).

88. Lombardi F, Taricco M, De Tanti A, Telaro E, Liberati A. Sensory stimulation for brain injured individuals in coma or vegetative state. Cochrane Database Syst. Rev. (2), CD001427 (2002).

89. Phillips CL, Bruno MA, Maquet P et al. 'Relevance vector machine' consciousness classifier applied to cerebral metabolism of vegetative and locked-in patients. Neuroimage 56(2), 797-808 (2011).

90. Larriviere D, Bonnie RJ. Terminating artificial nutrition and hydration in persistent vegetative state patients: current and proposed state laws. Neurology 66(11), 1624-1628 (2006). 